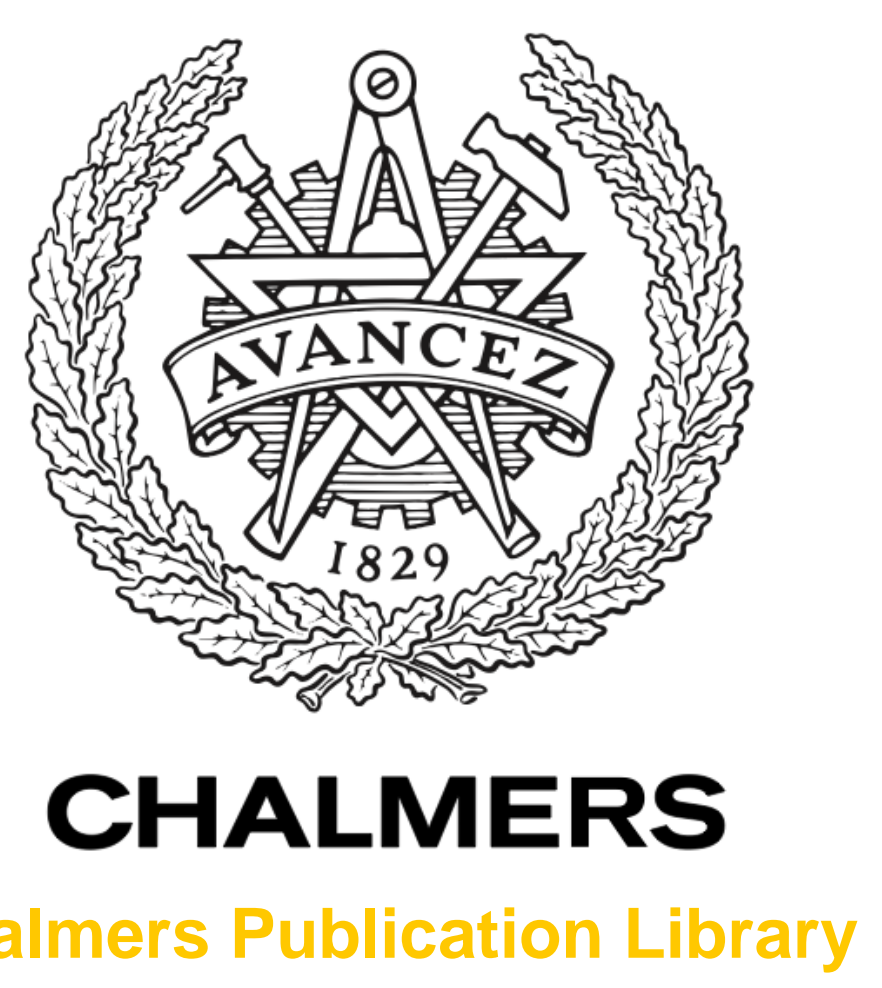

Chalmers Publication Library

\author{
Mixed-Mode Sensitivity Analysis of a Combined Differential and Common Mode \\ Active Receiving Antenna Providing Near-Hemispherical Field-of-View Coverage
}

This document has been downloaded from Chalmers Publication Library (CPL). It is the author's version of a work that was accepted for publication in:

IEEE Transactions on Antennas and Propagation (ISSN: 0018-926X)

Citation for the published paper:

Prinsloo, D. ; Maaskant, R. ; Ivashina, M. (2014) "Mixed-Mode Sensitivity Analysis of a Combined Differential and Common Mode Active Receiving Antenna Providing NearHemispherical Field-of-View Coverage". IEEE Transactions on Antennas and Propagation, vol. 62(8), pp. 3951-3961.

http://dx.doi.org/10.1109/tap.2014.2322896

Downloaded from: http://publications.lib.chalmers.se/publication/203296

Notice: Changes introduced as a result of publishing processes such as copy-editing and formatting may not be reflected in this document. For a definitive version of this work, please refer to the published source. Please note that access to the published version might require a subscription. 


\title{
Mixed-Mode Sensitivity Analysis of a Combined Differential and Common Mode Active Receiving Antenna Providing Near-Hemispherical Field-of-View Coverage
}

\author{
D. S. Prinsloo Student Member, IEEE, R. Maaskant Senior Member, IEEE, M. V. Ivashina Senior Member, IEEE, \\ and P. Meyer Member, IEEE
}

\begin{abstract}
A theoretical framework for a mixed differential and common mode sensitivity analysis of active receiving antennas is presented, which includes the derivation of a novel set of noise parameters for dual-mode balanced amplifiers. The analysis is applied to an example of a mixed-mode active wire antenna design, consisting of an integrated monopole and dipole structure. Results of numerical simulations and experimental measurements are presented which show that, for a single-polarized design, the judicious use of both differential and common modes enables the field-of-view coverage to be extended over the entire hemisphere with a variation in receiving sensitivity of less than $3 \mathrm{~dB}$ in the $E$-plane.
\end{abstract}

Index Terms-Receiving antennas, sensitivity, active antennas, differential amplifiers, microwave circuits.

\section{INTRODUCTION}

$\mathbf{T}$ HE vast majority of present-day receiving antennas are balanced in nature and therefore require baluns for use with Single-Ended (SE) Low-Noise Amplifiers (LNAs). The Common Mode (CM) component of the signal in such a system is then rejected by the antenna+balun and/or differential LNA, so that it suffices to optimize the antenna Differential Mode (DM) radiation and impedance characteristics only. However, baluns compromise compactness and increase ohmic losses, thereby reducing the antenna signal-to-noise ratio [1], [2].

Direct differential feeding obviates the use of baluns and represents a potential low-loss, low-cost antenna topology which allows for high integration with differential front-end electronics. Differential low-noise amplifiers (dLNAs) have therefore become increasingly popular amongst the microwave community during the last years [3]-[5]. However, the absence of baluns removes the suppression of $\mathrm{CM}$ signals in the system, and therefore an accurate analysis and design of these active antennas require proper handling of both common and

Manuscript received September 03, 2013

This work is based upon research supported by the South African Research Chairs Initiative of the Department of Science and Technology and the National Research Foundation, as well as the Swedish VR and VINNOVA funding agencies.

D. S. Prinsloo and P. Meyer are with the Department of Electrical and Electronic Engineering at the University of Stellenbosch, Stellenbosch, South Africa (e-mail: 16238087@sun.ac.za, pmeyer@sun.ac.za).

R. Maaskant and M. V. Ivashina are with the Signals and Systems Department at Chalmers University of Technology, Gothenburg, Sweden (email: rob.maaskant@chalmers.se, marianna.ivashina@chalmers.se). differential modes of the antenna. Furthermore, an understanding of how these modes propagate through the entire receiver system is crucial; for instance, it has been observed that CM signals can have a detrimental effect on the DM antenna performance when these undesired CM signals are excited at certain frequencies [6]-[9].

In this paper, we propose a novel dual-mode antenna design intrinsically supporting both the DM and CM signals, thereby creating an additional beamformer degree-of-freedom for improving the antenna impedance and radiation characteristics. The dual-mode antenna is based on a dipole combined with a parasitic monopole antenna to effectively exploit - rather than reject - the $\mathrm{CM}$ response. It should be noted that the associated common and differential mode patterns are dissimilar to the difference and sum patterns in monopulse radar tracking systems, where one typically employs a pair of identical antennas [10]. In wireless communication systems, the combination of a dipole and monopole into a single antenna element provides antenna diversity that can improve the reliability of the system in rich isotropic multipath (RIMP) environments [11], [12]. Furthermore, by combining the CM and DM patterns with arbitrary complex-valued weights, the beam may potentially be steered electronically over the entire hemisphere [13]. This extended scan range is of significant importance for the next generation radio telescopes requiring full sky surveys through the use of wide-scan phased array antenna systems [14], [15]. It is worth mentioning that S. Hay has shown that the aperture efficiency of the Australian Checkerboard phased array feed increased by 10-20 percent when all the outputs of pairs of SE LNAs making up the differential LNAs were beamformed individually, even though the antenna elements in that system were aimed to suppress CM signals [16].

Low-noise design for dense antenna arrays is in general a complex procedure due to the presence of mutual coupling between array elements [17]. Moreover, the introduction of an antenna element utilizing both common and differential modes adds extra complexity, as its design would require the knowledge of a full noise model, including all four noise parameters for both DM and CM cases, a full $S$-parameter description for both modes, as well as both DM and CM active impedances over a range of frequencies and scan angles.

While Mixed-Mode (MM) $S$-parameter theory is well es- 
tablished [18], the work on the MM noise performance of differential active antennas has been limited to the reporting of the presence of CM associated noise effects [6], [7]. This paper therefore presents a theoretical framework that can be used for the analysis and experimental characterization of receiving sensitivity of active antenna elements utilizing both $\mathrm{CM}$ and DM excitation. The framework includes a novel MM formulation of the standard noise parameters and extends the equivalent system representation of SE active array antennas, as proposed by [19], to the MM case.

The developed model has been validated numerically and through measurements, both by using a SE modeling approach. Note that the practical noise characterization of dLNAs, nonetheless, remains a challenging task because: (i) standard measurement techniques apply to two-port devices only, and; (ii) new methods for modeling and experimental characterization of the multi-port noise behavior reported thus far are limited to SE antenna-amplifier combinations with uncorrelated noises sources [3]-[5], [20]-[23].

The MM model introduced in this paper is applied to the newly proposed dual-mode antenna, showing that the receiving sensitivity increases significantly over the Field-of-View (FoV) relative to the purely differentially excited case.

\section{MiXEd-Mode Formulations}

\section{A. Mixed-Mode Antenna Analysis}

The operation of the proposed dual-mode antenna is illustrated in Fig. 1. The antenna consists of a dipole over ground and a parasitic monopole antenna integrated with a balanced transmission line feed. When excited differentially, no current is present on the monopole, creating a pure dipole-over-ground radiation pattern. In the case of a $\mathrm{CM}$ excitation, the dipole arms are excited in-phase and the monopole out-of-phase with respect to the dipole arms, realizing a near pure monopole radiation pattern.

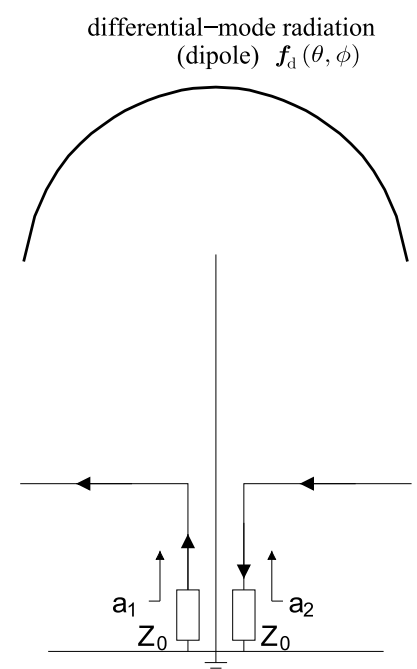

(a)

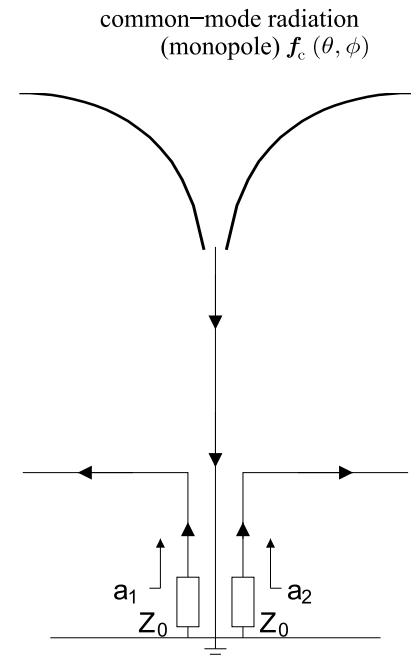

(b)
Fig. 1. Operation principle of the considered dual-mode antenna: (a) differential mode (dipole) radiation; (b) common mode (monopole) radiation.
When excited by two SE wave sources, two Embedded Element Patterns (EEPs) $\boldsymbol{f}_{1}(\theta, \phi)$ and $\boldsymbol{f}_{2}(\theta, \phi)$ result, and a $\mathrm{SE}$ two-port description is obtained in the form of the $S$-matrix $\mathrm{S}_{\mathrm{SE}}^{\text {ant }}$. Due to linearity, the DM and CM patterns and $S$-matrix are obtained by superposition of the SE excitations, to yield $\boldsymbol{f}_{\mathrm{d}}(\theta, \phi), \boldsymbol{f}_{\mathrm{c}}(\theta, \phi)$, and $\mathbf{S}_{\mathrm{MM}}^{\text {ant }}$ as:

$$
\begin{aligned}
& \boldsymbol{f}_{\mathrm{d}}(\theta, \phi)=\frac{1}{\sqrt{2}}\left[\boldsymbol{f}_{1}(\theta, \phi)-\boldsymbol{f}_{2}(\theta, \phi)\right] \\
& \boldsymbol{f}_{\mathrm{c}}(\theta, \phi)=\frac{1}{\sqrt{2}}\left[\boldsymbol{f}_{1}(\theta, \phi)+\boldsymbol{f}_{2}(\theta, \phi)\right],
\end{aligned}
$$

and

$$
\mathbf{S}_{\mathrm{MM}}^{\mathrm{ant}}=\left[\begin{array}{ll}
S_{\mathrm{dd}}^{\mathrm{ant}} & S_{\mathrm{dc}}^{\mathrm{ant}} \\
S_{\mathrm{cd}}^{\mathrm{ant}} & S_{\mathrm{cc}}^{\mathrm{ant}}
\end{array}\right],
$$

where from [18]

$$
\begin{aligned}
& S_{\mathrm{dd}}^{\text {ant }}=\left(S_{11}^{\mathrm{ant}}-S_{12}^{\mathrm{ant}}-S_{21}^{\mathrm{ant}}+S_{22}^{\mathrm{ant}}\right) / 2 \\
& S_{\mathrm{dc}}^{\text {ant }}=\left(S_{11}^{\mathrm{ant}}+S_{12}^{\mathrm{ant}}-S_{21}^{\mathrm{ant}}-S_{22}^{\mathrm{ant}}\right) / 2 \\
& S_{\mathrm{cd}}^{\text {ant }}=\left(S_{11}^{\mathrm{ant}}-S_{12}^{\mathrm{ant}}+S_{21}^{\mathrm{ant}}-S_{22}^{\mathrm{ant}}\right) / 2 \\
& S_{\mathrm{cc}}^{\text {ant }}=\left(S_{11}^{\mathrm{ant}}+S_{12}^{\mathrm{ant}}+S_{21}^{\mathrm{ant}}+S_{22}^{\mathrm{ant}}\right) / 2 .
\end{aligned}
$$

Using the complex-valued beamforming weights $w_{\mathrm{d}}(\theta, \phi)$ and $w_{\mathrm{c}}(\theta, \phi)$ for the DM and CM EEPs, the receiving sensitivity can be optimized over the FoV as explained in Sec. III. While the mechanism of combining weighted antenna patterns to achieve a new pattern follows that of standard arrays, the approach presented here differs in the fact that the array elements are collocated and the EEPs which are combined are not the same. This allows the MM receiver to exploit the different $\mathrm{DM}$ and $\mathrm{CM}$ element patterns to achieve an improved FoV coverage.

\section{B. Mixed-Mode Circuit Analysis}

The active antenna system comprises a balanced Differential Low-Noise Amplifier (dLNA), whose mathematical model involves the formulation of the MM $S$-parameters and an equivalent set of MM noise parameters. The combined antennaamplifier model, in turn, enables the receiving sensitivity to be modeled as explained in Sec. III.

The theory presented in [18] is used to derive the MM $S$ parameters of the balanced dLNA. Fig. 2 shows two SE LowNoise Amplifiers (LNAs) with the corresponding SE incident and reflected power waves, and an equivalent LNA with MM incident and reflected waves.

The MM $S$-parameters can be derived from the SE ones using the transformation

$$
\mathbf{S}_{\mathrm{MM}}^{\mathrm{dLNA}}=\mathbf{M}_{\mathrm{S}}^{\mathrm{dLNA}} \mathbf{S}_{\mathrm{SE}}^{\mathrm{dLNA}}\left(\mathbf{M}_{\mathrm{S}}^{\mathrm{dLNA}}\right)^{-1}
$$

where the transformation matrix $\mathbf{M}_{\mathrm{S}}^{\mathrm{dLNA}}$ is defined as

$$
\mathbf{M}_{\mathrm{S}}^{\mathrm{dLNA}}=\frac{1}{\sqrt{2}}\left[\begin{array}{cccc}
1 & 0 & -1 & 0 \\
0 & 1 & 0 & -1 \\
1 & 0 & 1 & 0 \\
0 & 1 & 0 & 1
\end{array}\right]
$$




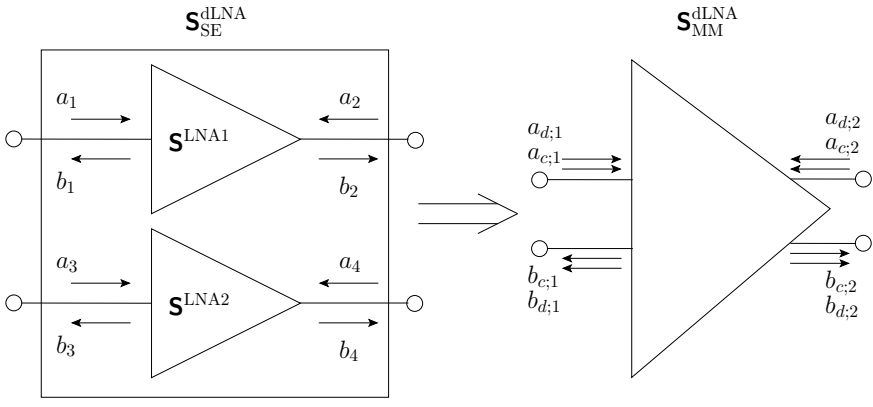

Fig. 2. Incident and reflected power waves at the SE and MM input and output ports of the corresponding LNAs.

It is useful to block-partition the MM scattering matrix as

$$
\mathbf{S}_{\mathrm{MM}}^{\mathrm{dLNA}}=\left[\begin{array}{l|l}
\mathbf{S}_{\mathrm{dd}}^{\mathrm{dLNA}} & \mathbf{S}_{\mathrm{dc}}^{\mathrm{dLNA}} \\
\hline \mathbf{S}_{\mathrm{cd}}^{\mathrm{dLNA}} & \mathbf{S}_{\mathrm{cc}}^{\mathrm{dLNA}}
\end{array}\right]
$$

where $\mathbf{S}_{\mathrm{dd}}^{\mathrm{dLNA}}, \mathbf{S}_{\mathrm{cc}}^{\mathrm{dLNA}}$, and $\left\{\mathbf{S}_{\mathrm{cd}}^{\mathrm{dLNA}}, \mathbf{S}_{\mathrm{dc}}^{\mathrm{dLNA}}\right\}$, denote the $2 \times 2 \mathrm{DM}$, CM, and cross-mode $S$-parameter matrices of the dLNA, respectively. For the balanced system considered in this analysis, the two SE LNAs are assumed to be identical and perfectly isolated, i.e. $\mathbf{S}^{\mathrm{LNA} 1}=\mathbf{S}^{\mathrm{LNA} 2}=\mathbf{S}^{\mathrm{LNA}}$, so that the four-port $\mathrm{SE}$ scattering matrix takes the form

$$
\mathbf{S}_{\mathrm{SE}}^{\mathrm{dLNA}}=\left[\begin{array}{cccc}
S_{11}^{\mathrm{LNA}} & S_{12}^{\mathrm{LNA}} & 0 & 0 \\
S_{21}^{\mathrm{LNA}} & S_{22}^{\mathrm{LNA}} & 0 & 0 \\
0 & 0 & S_{11}^{\mathrm{LNA}} & S_{12}^{\mathrm{LNA}} \\
0 & 0 & S_{21}^{\mathrm{LNA}} & S_{22}^{\mathrm{LNA}}
\end{array}\right]
$$

which, after the application of (4), renders the cross-terms $\mathbf{S}_{\mathrm{cd}}^{\mathrm{dLNA}}=\mathbf{S}_{\mathrm{dc}}^{\mathrm{dLNA}}=\mathbf{0}$ in (6).

In addition to the MM $S$-parameters, a corresponding set of MM equivalent noise parameters of the dLNA is required. The derivation of such a set of noise parameters is presented in the Appendix, and summarized in (8) for the case of two identical and isolated LNAs of which the noise contributions are uncorrelated. In (8), $R_{\mathrm{n}}, F_{\mathrm{min}}$, and $Y_{\mathrm{opt}}$ are the standard SE noise parameters.

\begin{tabular}{c|c} 
DM Noise Parameters & CM Noise Parameters \\
\hline$F_{\min }^{\mathrm{d}}=F_{\min }$ & $F_{\min }^{\mathrm{c}}=F_{\min }$ \\
$R_{\mathrm{n}}^{\mathrm{d}}=2 R_{\mathrm{n}}$ & $R_{\mathrm{n}}^{\mathrm{c}}=R_{\mathrm{n}} / 2$ \\
$Y_{\mathrm{opt}}^{\mathrm{d}}=Y_{\mathrm{opt}} / 2$ & $Y_{\mathrm{opt}}^{\mathrm{c}}=2 Y_{\mathrm{opt}}$
\end{tabular}

In general the LNAs in active antenna systems are fed by coupled transmission lines. The DM and CM characteristic impedances of a pair of coupled transmission lines can, respectively, be related to the odd mode $\left(Z_{0}^{\mathrm{o}}\right)$ and even mode $\left(Z_{0}^{\mathrm{e}}\right)$ characteristic impedances of each line, i.e., [18]

$$
Z_{0}^{\mathrm{d}}=2 Z_{0}^{\mathrm{o}}, \quad \text { and } \quad Z_{0}^{\mathrm{c}}=Z_{0}^{\mathrm{e}} / 2
$$

from which the equivalent SE characteristic impedance is calculated as [24]

$$
Z_{0}=\sqrt{Z_{0}^{\mathrm{o}} Z_{0}^{\mathrm{e}}}
$$

where $Z_{0}$ is the real-valued SE characteristic impedance. Assuming uncoupled transmission lines results in $Z_{0}^{\mathrm{o}}=Z_{0}^{\mathrm{e}}=Z_{0}$.
Together with (8), and for identical SE LNAs, each with optimum source reflection coefficient $\Gamma_{\text {opt }}$, this results in

$$
\Gamma_{\mathrm{opt}}^{\mathrm{d}}=\Gamma_{\mathrm{opt}}^{\mathrm{c}}=\Gamma_{\mathrm{opt}}
$$

\section{Receiving Sensitivity Modeling}

Modeling the receiving sensitivity requires a detailed description of the receiver noise. In [17] it is shown that higher sensitivity is achieved when the receivers are noise matched to the active reflection coefficient of a beamforming array rather than the passive reflection coefficient of the array antennas. This section applies a similar analysis to the dual-mode active antenna to solve both the SE and MM active reflection coefficients, effectively noise-decoupling the two channels (or modes). The noise analysis presented in [19] is then applied to the noise-decoupled receivers, of both the SE and MM representations, in order to evaluate the respective receiver noise temperatures. In the analysis to follow, $[p, q]$ denotes either the channels $[1,2]$ or the modes $[d, c]$, and $\left\{\mathbf{S}^{(p)}, \mathbf{S}^{(q)}\right\}$ represent the scattering parameters $\left\{\mathbf{S}^{\mathrm{LNA} 1}, \mathbf{S}^{\mathrm{LNA} 2}\right\}$ or $\left\{\mathbf{S}_{\mathrm{dd}}^{\mathrm{dLNA}}, \mathbf{S}_{\mathrm{cc}}^{\mathrm{dLNA}}\right\}$ for the SE and MM representations, respectively.

\section{A. Receiver Noise}

Fig. 3(a) shows the dual-mode active antenna connected to two SE LNAs with complex beamforming weights $w_{1}$ and $w_{2}$ applied to the respective channels. The correlated noise waves emanating from the input and output of each LNA are denoted by $\left[c_{1}^{(1)}, c_{2}^{(1)}\right]$ and $\left[c_{1}^{(2)}, c_{2}^{(2)}\right]$, respectively. The equivalent $\mathrm{MM}$ representation of the active dual-mode antenna is shown in Fig. 3(b) with the respective DM and CM complex beamforming weights $w_{d}$ and $w_{c}$ applied to each mode. In the MM representation each antenna represents one of the two modes and their coupling is defined by the cross-mode antenna scattering parameters. The equivalent DM and CM correlated input and output noise waves are denoted by $\left[c_{1}^{(\mathrm{d})}, c_{2}^{(\mathrm{d})}\right]$ and $\left[c_{1}^{(\mathrm{c})}, c_{2}^{(\mathrm{c})}\right]$, respectively.

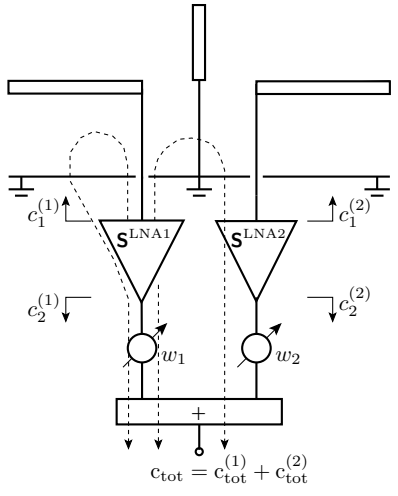

(a)

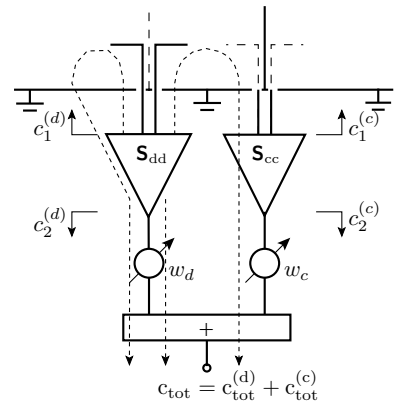

(b)
Fig. 3. Propagation paths of the (a) single-ended noise waves and (b) mixedmode noise waves in the coupled dual-mode active antenna.

The active reflection coefficients of the individual channels/modes are solved by expressing the noise wave at the output of the ideal power combiner - due to the respective 
channel/mode - as the superposition of three noise wave contributions, i.e.,

$$
\begin{aligned}
c_{\mathrm{tot}}^{(p)} & =\text { Direct Part }+ \text { Reflected Part }+ \text { Coupled Part } \\
& =w_{p} c_{2}^{(p)}+w_{p} c_{1}^{(p)} S_{p p}^{\text {ant }} S_{21}^{(p)}+w_{q} c_{1}^{(p)} S_{q p}^{\text {ant }} S_{21}^{(q)},
\end{aligned}
$$

For identical LNAs, with gains $S_{21}^{\mathrm{LNA}}$, the expression in (12) reduces to

$$
\begin{aligned}
c_{\mathrm{tot}}^{(p)} & =w_{p}\left[c_{2}^{(p)}+c_{1}^{(p)} S_{21}^{\mathrm{LNA}}\left(S_{p p}^{\mathrm{ant}}+\frac{w_{q}}{w_{p}} S_{q p}^{\mathrm{ant}}\right)\right] \\
& =w_{p}\left[c_{2}^{(p)}+c_{1}^{(p)} S_{21}^{\mathrm{LNA}} \Gamma_{\mathrm{act}}^{(p)}\right]
\end{aligned}
$$

from which it follows that for a reciprocal antenna, the active reflection coefficient of channel $p$ equates to $\Gamma_{\text {act }}^{(p)}=S_{p p}^{\text {ant }}+$ $\frac{w_{q}}{w_{p}} S_{p q}^{\text {ant }}$. Similarly, the active reflection coefficient of channel $q$ can be shown to equal $\Gamma_{\text {act }}^{(q)}=S_{q q}^{\text {ant }}+\frac{w_{p}}{w_{q}} S_{q p}^{\text {ant }}$. Using these active reflection coefficients the antenna can be represented as two noise-decoupled channels/modes, as illustrated in Fig 4.

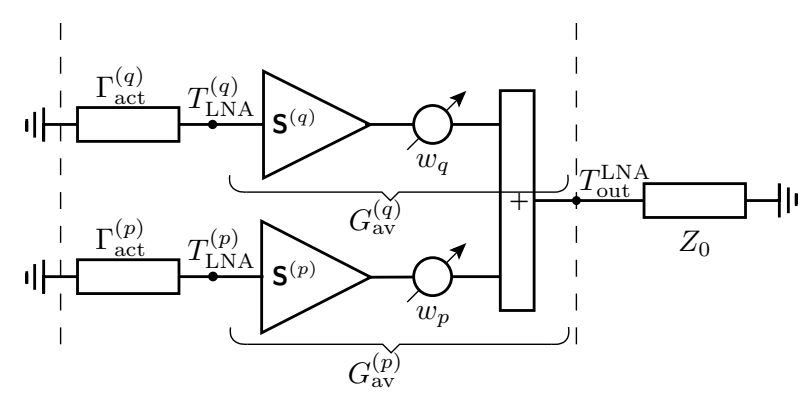

Fig. 4. Equivalent noise-decoupled representation of the dual-mode active antenna.

Fig. 4 can be used to calculate the equivalent input referred noise contributed by the receiver for both the SE and MM representations. The total noise contribution due to the LNAs is given by the weighted sum of the uncorrelated noise contributions

$$
T_{\mathrm{out}}^{\mathrm{LNA}}=G_{\mathrm{av}}^{(p)} T_{\mathrm{LNA}}^{(p)}+G_{\mathrm{av}}^{(q)} T_{\mathrm{LNA}}^{(q)},
$$

where the noise temperature $T_{\mathrm{LNA}}^{(\nu)}$ is referred to the input of channel/mode $\nu=p, q$ and is weighted by the respective available gain $G_{\mathrm{av}}^{(\nu)}$ - defined from the output of the antenna to the output of the ideal power combiner, i.e.,

$$
G_{\mathrm{av}}^{(\nu)}=\frac{1}{2}\left|S_{21}^{(\nu)}\right|^{2}\left|w_{\nu}\right|^{2}\left(1-\left|\Gamma_{\mathrm{act}}^{(\nu)}\right|^{2}\right), \quad \text { for } \quad \nu=p, q .
$$

The input referred noise temperature of each channel (or mode) is obtained using the well-known formula for noisy two-ports:

$$
T_{\mathrm{LNA}}^{(\nu)}=T_{\min }+\frac{4 R_{\mathrm{n}}^{(\nu)} T_{0}}{Z_{0}^{(\nu)}}\left[\frac{\left|\Gamma_{\mathrm{act}}^{(\nu)}-\Gamma_{\mathrm{opt}}\right|^{2}}{\left|1+\Gamma_{\mathrm{opt}}\right|^{2}\left(1-\left|\Gamma_{\mathrm{act}}^{(\nu)}\right|^{2}\right)}\right]
$$

for $\nu=p, q$, where $R_{\mathrm{n}}^{(\nu)}, T_{\min }, \Gamma_{\mathrm{opt}}$ are the noise parameters of the LNA, $T_{0}$ is the standard temperature $(290 \mathrm{~K})$ and $Z_{0}^{(\nu)}$ the real-valued characteristic impedance for the specific channel or mode.
Dividing (14) by the equivalent available gain of the LNAs yields the equivalent input referred noise of the receiver, i.e.,

$$
T_{\mathrm{eq}}=T_{\mathrm{out}}^{\mathrm{LNA}} / G_{\mathrm{av}}^{\mathrm{eq}},
$$

where

$$
\begin{aligned}
G_{\mathrm{av}}^{\mathrm{eq}}=\frac{1}{2}\left[\left|w_{p}\right|^{2}\left|S_{21}^{(p)}\right|^{2}\right. & \left(1-\left|\Gamma_{a c t}^{(p)}\right|^{2}\right) \\
& \left.+\left|w_{q}\right|^{2}\left|S_{21}^{(q)}\right|^{2}\left(1-\left|\Gamma_{a c t}^{(q)}\right|^{2}\right)\right] .
\end{aligned}
$$

Furthermore, for equal SE LNA gains, (17) reduces to

$$
T_{\mathrm{eq}}=\frac{\left|w_{p}\right|^{2}\left(1-\left|\Gamma_{\mathrm{act}}^{(p)}\right|^{2}\right) T_{\mathrm{LNA}}^{(p)}+\left|w_{q}\right|^{2}\left(1-\left|\Gamma_{\mathrm{act}}^{(q)}\right|^{2}\right) T_{\mathrm{LNA}}^{(q)}}{\left|w_{p}\right|^{2}\left(1-\left|\Gamma_{\mathrm{act}}^{(p)}\right|^{2}\right)+\left|w_{q}\right|^{2}\left(1-\left|\Gamma_{\mathrm{act}}^{(q)}\right|^{2}\right)} .
$$

\section{B. Receiving Sensitivity}

The receiving sensitivity of the active antenna is defined as the ratio of the effective antenna area to the total system noise temperature, i.e., $A_{\text {eff }} / T_{\text {sys }}$, where the well-known reciprocity relation $A_{\text {eff }}=(4 \pi)^{-1} \lambda^{2} G$ relates the effective area to the antenna gain $G(\Omega)$, defined as the ratio of the radiated power per solid angle, $P(\Omega)$, to the accepted input power of the antenna, $P_{\text {in }}$. Here, $P(\Omega)$ can be expressed in terms of the weighted EEPs for wave excitations, i.e., $P(\Omega)=(2 \eta)^{-1}\left|w_{p} \boldsymbol{f}_{p}(\Omega)+w_{q} \boldsymbol{f}_{q}(\Omega)\right|^{2}$, with $\eta$ denoting the free-space impedance. The effective area is therefore expressed as

$$
A_{\text {eff }}=\frac{\lambda^{2}}{2 \eta} \frac{\left|w_{p} \boldsymbol{f}_{p}(\Omega)+w_{q} \boldsymbol{f}_{q}(\Omega)\right|^{2}}{P_{\text {in }}},
$$

where $\lambda$ denotes the wavelength, and $P_{\text {in }}=\mathbf{w}^{H}\left(\mathbf{I}-\mathbf{S}^{H} \mathbf{S}\right) \mathbf{w}$ is the total accepted input power, with $\mathbf{I}$ the $2 \times 2$ identity matrix, and where $\{\mathbf{w}, \mathbf{S}\}$ either denotes $\left\{\left[w_{1}, w_{2}\right]^{T}, \mathbf{S}_{\mathrm{SE}}^{\text {ant }}\right\}$ or $\left\{\left[w_{\mathrm{d}}, w_{\mathrm{c}}\right]^{T}, \mathbf{S}_{\mathrm{MM}}^{\text {ant }}\right\}$ for the SE and $\mathrm{MM}$ representations, respectively.

Generally, $T_{\text {sys }}$ consists of three main contributions: (i) the spillover noise; (ii) the noise due to dissipation losses of the antenna, and; (iii) the noise due to the LNAs which depend on the noise properties of the LNAs and active reflection coefficients at the antenna ports [19]. Since the focus of this paper is on the MM receiver characterization of an active dualmode antenna, only the latter noise contribution is taken into account, so that $T_{\text {sys }}=T_{\text {eq }}$, where the equivalent input referred noise of the receiver, $T_{\text {eq }}$, is calculated using (19). Hence, the final receiving sensitivity is given by

$$
\frac{A_{\mathrm{eff}}}{T_{\mathrm{sys}}}=\frac{\lambda^{2}}{2 \eta T_{\mathrm{eq}}} \frac{\left|w_{p} \boldsymbol{f}_{p}(\Omega)+w_{q} \boldsymbol{f}_{q}(\Omega)\right|^{2}}{\mathbf{w}^{H}\left(\mathbf{I}-\mathbf{S}^{H} \mathbf{S}\right) \mathbf{w}} .
$$

\section{Dual-Mode Antenna Design with Large FIELD-OF-VIEW COVERAGE}

In [13], a simple proof-of-concept dual-mode antenna, consisting of a balanced microstrip transmission line feed and an inverted-V dipole on the top layer of a Rogers Duroid RO4003 substrate, and a monopole which extends out from a ground plane on the bottom layer, was demonstrated theoretically and 
verified experimentally. Whilst demonstrating the viability of a dual-mode structure, this antenna exhibited limited bandwidth and significant sensitivity variation over the FoV. The present paper proposes an improved dual-mode antenna design exhibiting a smaller gain variation and receiving sensitivity variation over the FoV.

\section{A. Dual-Mode Antenna Design}

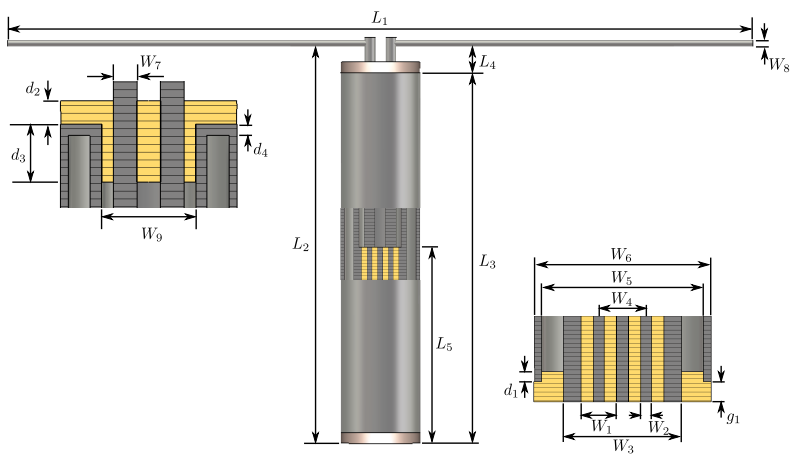

Fig. 5. Cylindrical dual-mode antenna design with cut planes at the bottom, middle (semi-rigid coaxial to air-core twinaxial transition) and top of the antenna feed.

TABLE I

CYLINDRICAL DUAL-MODE ANTENNA DESIGN PARAMETERS.

\begin{tabular}{c|c|l} 
Parameter & Value $[\mathrm{mm}]$ & Description \\
\hline$L_{1}$ & 141 & Dipole length \\
$L_{2}$ & 75 & Dipole height \\
$L_{3}$ & 70 & Feed/Monopole height \\
$L_{4}$ & 5 & Dipole-Monopole separation \\
$L_{5}$ & 35 & Height of twinaxial transition \\
$W_{1}$ & 3 & Coaxial dielectric diameter \\
$W_{2}$ & 0.91 & Coaxial conductor diameter \\
$W_{3}$ & 10 & Feed ground shield outer diameter \\
$W_{4}$ & 5 & Dipole/Feed line separation \\
$W_{5}$ & 13.66 & Monopole sleeve inner diameter \\
$W_{6}$ & 15 & Monopole sleeve outer diameter \\
$W_{7}$ & 2 & Twinaxial inner conductor diameter \\
$W_{8}$ & 1.15 & Dipole arm diameter \\
$W_{9}$ & 8 & Twinaxial ground shield inner diameter \\
$d_{1}$ & 1 & Monopole Teflon support \\
$d_{2}$ & 2 & Twinaxial conductor Teflon support \\
$d_{3}$ & 5 & Twinaxial conductor Teflon support \\
$d_{4}$ & 1 & Monopole-Feed ground cap thickness \\
$g_{1}$ & 2 & Monopole-Ground plane gap height
\end{tabular}

The improved dual-mode antenna is realized by combining a cylindrical dipole and monopole element with a single twinaxial feed, as depicted in Fig. 5. The cylindrical dualmode antenna is realized by a single balanced transmission line feeding a dipole element - where each of the two center conductors is connected to one of the dipole arms. Rather than extending the monopole from the ground conductor of the transmission line, as is done in the planar design [13], the monopole is realized by folding the ground conductor back towards the ground plane - leaving a small gap between the monopole and the ground shield of the feed as well as the ground plane $\left(g_{1}\right)$. To keep the monopole sleeve in place, a small Teflon spacer is placed at the foot of the antenna. The antenna is excited through two $3 \mathrm{~mm}$ semi-rigid coaxial cables extending midway into the antenna feed, from which point the center conductors of the semi-rigid coaxial cables extend further to form an air-core twinaxial transmission line. To ensure the stability of the center conductors of the twinaxial transmission line, another Teflon spacer is placed at the top of the monopole sleeve. Table I summarizes the antenna design parameters illustrated in Fig. 5.

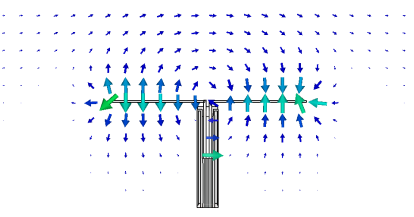

(a)

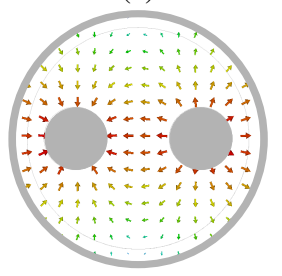

(c)

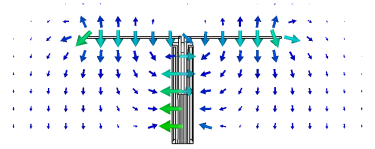

(b)

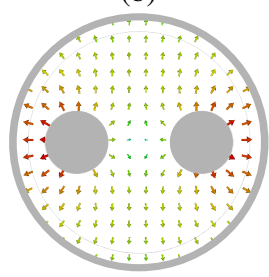

(d)
Fig. 6. Simulated electric field distributions (a) DM antenna near field (b) $\mathrm{CM}$ antenna near field (c) DM port excitation (d) CM port excitation.

Fig. 6 illustrates the MM operation of the antenna topology, shown in Fig. 5, simulated over an infinite ground plane using CST. The DM field distribution in the balanced transmission line feed [c.f. Fig. 6(c)] is seen to excite the dipole arms out of phase, realizing a typical dipole radiated electric near field [c.f. Fig. 6(a)]. A CM excitation realizes the field distribution of the feed as depicted in Fig. 6(d) and is shown to excite the dipole arms in-phase, but out-of-phase with respect to the monopole sleeve, resulting in a monopole-like radiated electric near field [c.f. Fig. 6(b)].

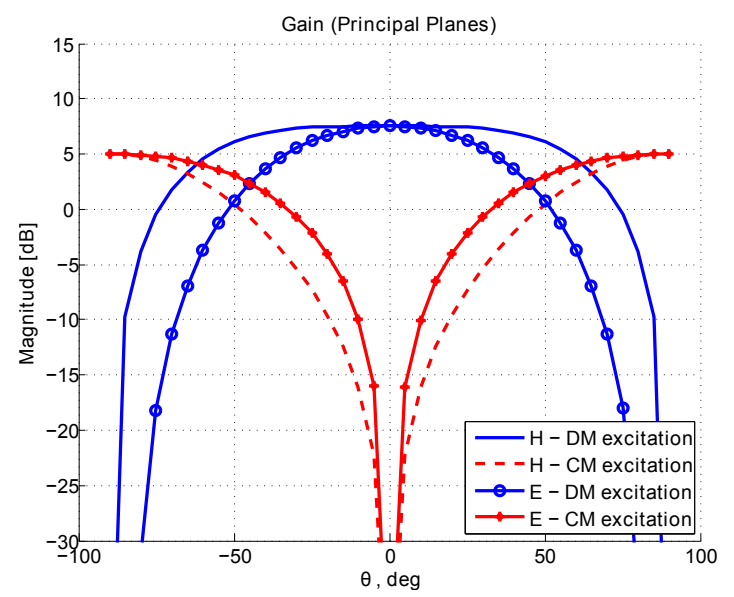

Fig. 7. Differential and common mode $E$-plane and $H$-plane gain simulated at $1 \mathrm{GHz}$.

Fig. 7 shows the normalized $E$ - and $H$-plane MM gain patterns resulting from the DM and CM excitations depicted in Fig. 6. The curves in Fig. 7 clearly illustrate that a typical dipole-over-ground radiation pattern is realized by a DM excitation, and that a $\mathrm{CM}$ excitation results in a monopoleover-ground radiation pattern. 


\section{B. Experimental Verification}

In order to measure the performance of the antenna, the design illustrated in Fig. 5 is placed in the center of a circular ground plane with a diameter of $500 \mathrm{~mm}$, and excited through two $3 \mathrm{~mm}$ semi-rigid coaxial cables shown in Fig. 8.

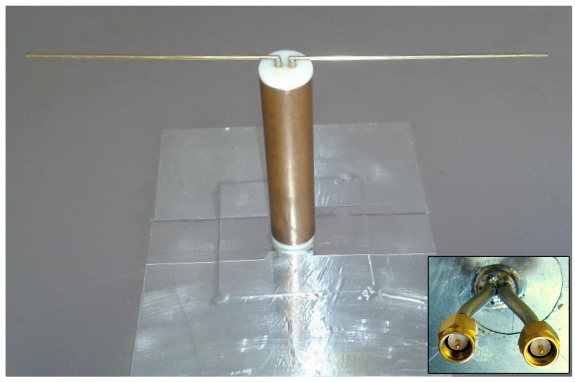

Fig. 8. Dual-mode antenna on finite circular ground plane with $3 \mathrm{~mm}$ semirigid coaxial feeds.

Using (3a) and (3d), the DM and CM input reflection coefficients can be solved from the measured SE S-parameters of the antenna, respectively. The graph in Fig. 9 compares the measured MM input reflection coefficients to the simulated results obtained using MM excitations in CST. Fig. 9 shows that the measurements agree very well with the simulated response, with both differential and common modes matched at the center frequency of $1 \mathrm{GHz}$. Also shown in Fig. 9 is the measured isolation between the DM and CM excitations calculated using (3b). It is seen that an isolation below $-30 \mathrm{~dB}$ is achieved.

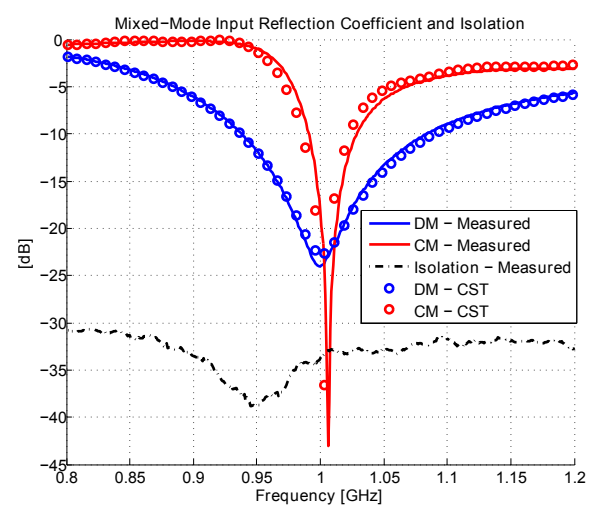

Fig. 9. Measured and simulated differential and common mode input reflection coefficients and isolation.

Similar to the MM reflection coefficients, the DM and CM radiation patterns are obtained by measuring the SE radiation patterns of the antenna and applying (1a) and (1b), respectively [13]. The co-polar MM radiation patterns measured and simulated, at $1 \mathrm{GHz}$, in both principal planes are compared in Fig. 10.

The measured differential and common mode radiation patterns are seen to be in good agreement with the simulated response [c.f. Fig. 10] in both principal planes. It should be noted that the differences in the common mode pattern of the measured antenna, shown in Fig. 10, and the radiation patterns depicted in Fig. 7 can be attributed to the finite ground plane used for the physical antenna. Regardless of these differences,

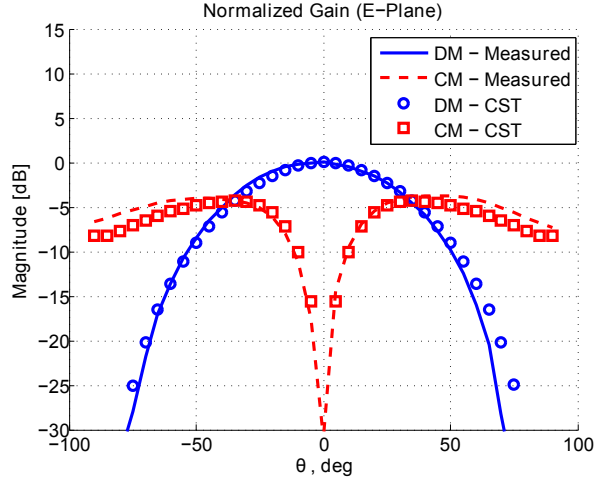

(a)

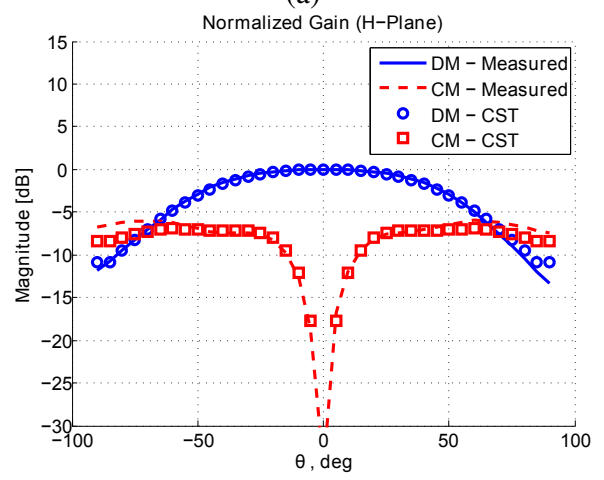

(b)

Fig. 10. Measured and simulated co-polar differential and common mode radiation patterns (a) $E$-plane and (b) $H$-plane.

the measurements still illustrate the dipole- and monopolelike radiation characteristics of the antenna resulting from differential and common mode excitations, respectively.

\section{Dual-Mode Active Receiver}

A schematic representation of the MM active receiver analyzed in this section is shown in Fig. 3(b), where the active antenna element is realized using the cylindrical antenna design in Fig. 5, placed on an infinite ground plane and connected to two identical SE LNAs. For the purpose of this investigation, an LNA model with typical noise-parameter values for $F_{\min }$ and $R_{\mathrm{n}}$ is chosen, while $\Gamma_{\mathrm{opt}}$ is selected to provide a noise-match to the passive differential antenna input impedance. In addition, idealized $S$-parameters are used, as shown in Table II. The use of these ideal values does not affect the conclusions of the analysis.

TABLE II

SINGLE-ENDED LNA NOISE AND $S$-PARAMETERS.

\begin{tabular}{c|l} 
Noise Parameters & \multicolumn{1}{c}{$S$-Parameters } \\
\hline$T_{\min }=37 \mathrm{~K}$ & $S_{21}=20$ \\
$R_{\mathrm{n}}=3 \Omega$ & $S_{12}=0$ \\
$\Gamma_{\mathrm{opt}}=S_{\mathrm{ant}}^{\text {dd }}$ & $S_{11}=S_{22}=0$
\end{tabular}

In order to analyze the MM sensitivity, the SE parameters shown in table II are transformed into the equivalent MM $S$ and noise parameters using (4) and (8), respectively. 


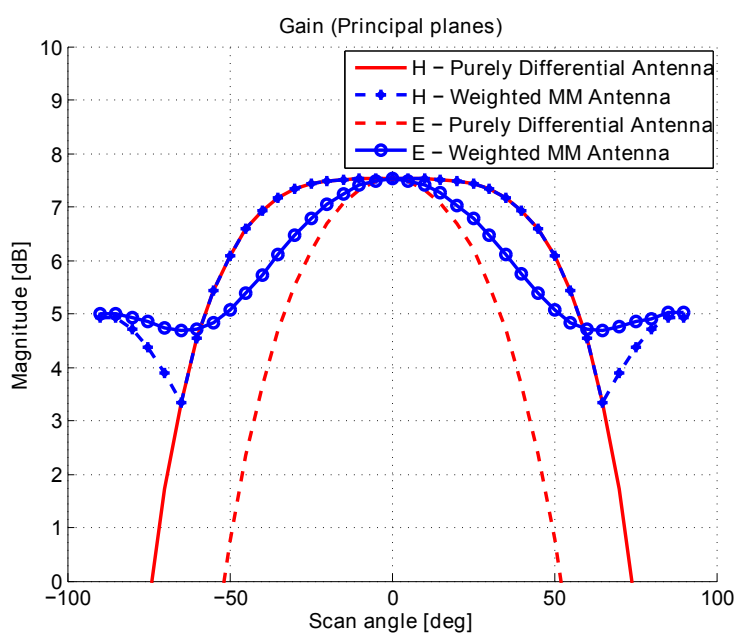

Fig. 11. Weighted MM $E$ - and $H$-plane gain optimized for each scan angle compared to purely differential excitation.

\section{A. Effective Area}

By solving the MM beamforming weight vector $\mathbf{w}=$ $\left[w_{\mathrm{d}}, w_{\mathrm{c}}\right]^{T}$ for maximum sensitivity at each scan angle [25], the performance of a MM receiving element - allowing for both DM and CM propagation - can be compared to that of a conventional purely differential receiver. Fig. $11 \mathrm{com}$ pares the MM antenna gain, with the respective MM weights optimized for maximum sensitivity using the DM and CM EEPs predicted by CST, to the antenna gain realized for a purely differential excitation, in both the $E$ - and $H$-planes. The curves show the antenna gain at $1 \mathrm{GHz}$, and clearly indicate the improved FoV realized by allowing CM propagation. With the weighted MM antenna gain deviating by less than $3 \mathrm{~dB}$ in the $E$-plane and by only $4 \mathrm{~dB}$ in the $H$-plane, this antenna effectively realizes near hemispherical FoV coverage.

\section{B. Equivalent Noise Temperature}

Since this increase in effective area is maximized by applying complex-valued beamforming weights to each of the two modes, the equivalent noise contribution of the MM receiver should be computed using the active reflection coefficient as discussed in Sec. III, rather than the passive reflection coefficient considered in the purely differential receiver. Using $\mathbf{S}_{\mathrm{MM}}^{\text {ant }}$ as calculated by CST, the noise contributed to the receiver noise temperature due to the weighted differential and common mode is shown for both the $E$ - and the $H$-planes in Fig. 12 .

Fig. 12 indicates that equivalent noise contributed by the purely differential receiver remains constant at the minimum noise temperature of the SE LNA listed in Table II. This is to be expected given that the optimum source reflection coefficient of the LNA equals the passive differential reflection coefficient of the antenna.

For the MM noise contribution, first consider the $E$-plane noise contribution in Fig. 12. The weighted MM antenna gain in Fig. 11 ( $E$-plane) is seen to be almost equal to a purely DM excitation for scan angles of $0^{\circ} \leq \theta_{\text {scan }} \leq 20^{\circ}$ from zenith. For these scan angles the DM weights are found to be significantly more dominant than the CM weights, resulting in
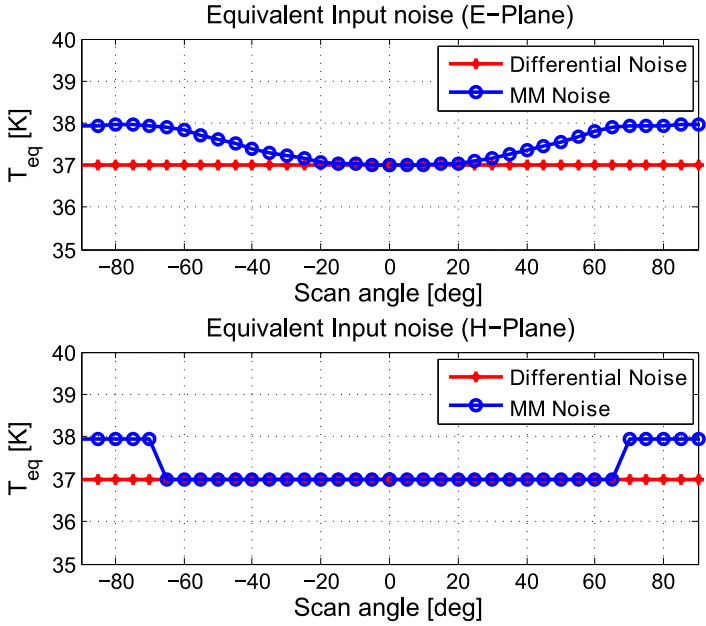

Fig. 12. Purely differential and MM equivalent input noise temperature in both the $E$ - and $H$-planes.

an active differential reflection coefficient close to the passive reflection coefficient of the antenna. Together with low CM weight values, this results in the MM receiver being noise matched in the $E$-plane at $0^{\circ} \leq \theta_{\text {scan }} \leq 20^{\circ}$ from zenith. For $20^{\circ} \leq \theta_{\text {scan }} \leq 50^{\circ}$ from zenith, the MM antenna gain is seen to be greater than that of the the purely DM receiver. This increase in gain is due to the addition of CM propagation realized through an increase in the complex $\mathrm{CM}$ weight values. Since the LNAs are noise matched to the passive differential impedance of the antenna, the $\mathrm{CM}$ noise mismatch results in a larger $\mathrm{CM}$ noise contribution. Considering Fig. 12 at $20^{\circ} \leq \theta_{\text {scan }} \leq 50^{\circ}$ from zenith, this $\mathrm{CM}$ noise is seen to increase the MM equivalent input noise proportionally to the $\mathrm{CM}$ weight values applied at these scan angles. The $E$-plane gain in Fig. 11 shows that the MM antenna gain is dominated by a $\mathrm{CM}$ excitation at scan angles $50^{\circ} \leq \theta_{\text {scan }} \leq 90^{\circ}$ from zenith, realizing CM weights that are substantially larger than the DM weights, and in turn resulting in CM noise dominating the MM equivalent input noise. This behavior is noted in the increase of the equivalent noise temperature of the $\mathrm{MM}$ receiver in Fig. 12.

Next, consider the $H$-plane antenna gain patterns and equivalent receiver noise contribution shown in Figs. 11 and 12, respectively. Fig. 11 shows that the weighted MM gain equals the gain of the purely differentially excited antenna for scan angles $0^{\circ} \leq \theta_{\text {scan }} \leq 60^{\circ}$ from zenith. The $\mathrm{CM}$ weights at these scan angles are therefore nearly negligible compared to the DM weights, and hence the equivalent noise contribution of the weighted MM receiver equals that of the DM receiver as shown in Fig. 12. For scan angles $60^{\circ} \leq \theta_{\text {scan }} \leq 90^{\circ}$ from zenith, the $H$-plane $\mathrm{MM}$ antenna gain is realized primarily due to a $\mathrm{CM}$ excitation, resulting in dominating $\mathrm{CM}$ weights. Analogous to the $E$-plane, the increase in CM weights at these larger scan angles are seen to result in CM noise dominating the MM equivalent noise contribution in Fig. 12.

\section{Receiving Sensitivity}

As indicated in (21), the receiving sensitivity is approximated by only accounting for the noise contributed by the 
LNA. The normalized sensitivity, in both the $E$ - and $H$ planes, of the MM receiver is compared to the sensitivity of a conventional differential receiver in Figs. 13(a) and (b), respectively.

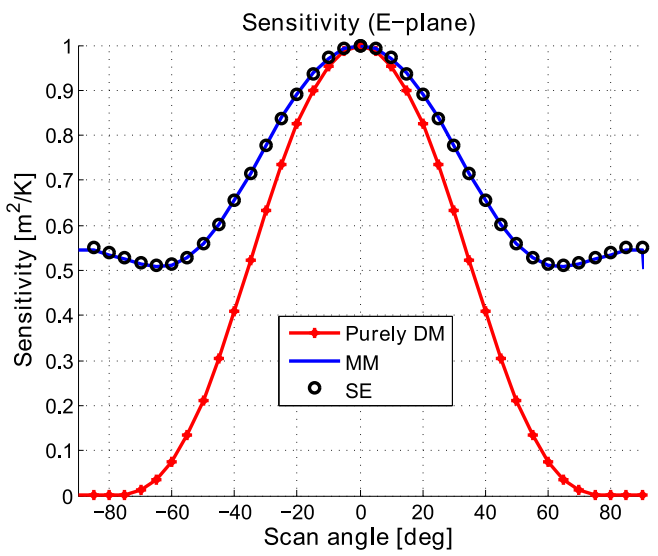

(a)

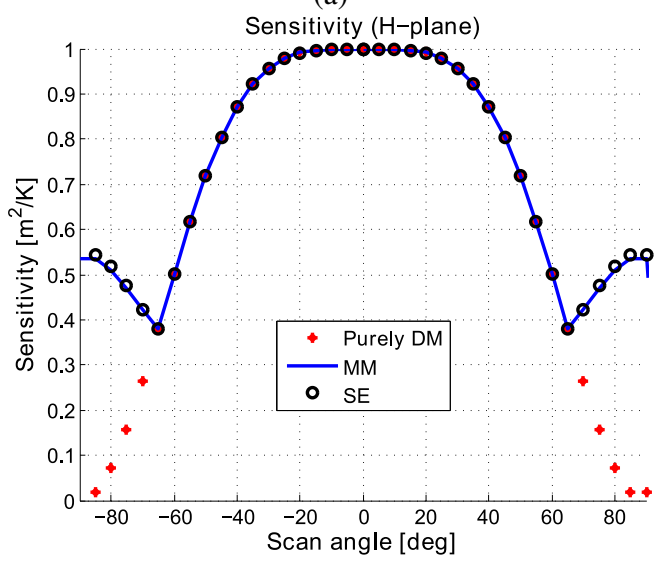

(b)

Fig. 13. Purely differential and dual-mode sensitivity (a) E-plane and (b) $H$-plane.

Fig. 13 indicates that, regardless of the increase in the equivalent noise temperature attributed to the $\mathrm{CM}$ present in the $\mathrm{MM}$ receiver, the utilization of $\mathrm{CM}$ propagation can result in an increase in the sensitivity over the entire FoV coverage when compared to the conventional receivers where $\mathrm{CM}$ is completely rejected - realizing a variation in sensitivity of less than $50 \%$ in the $E$ - and $60 \%$ in the $H$-plane. A comparison between the variation in sensitivity in Fig. 13 and the gain variation depicted in Fig. 11 shows that the variation in sensitivity corresponds to the gain variation in both planes.

As a final validation of the MM receiver model presented in this paper, Fig. 13 shows that the MM sensitivity analysis produces the same result obtained when analyzing the antenna and receiver using the equivalent $\mathrm{SE} S$-matrix $\mathrm{S}_{\mathrm{SE}}^{\text {ant }}$ and EEPs $\boldsymbol{f}_{1}(\theta, \phi)$ and $\boldsymbol{f}_{2}(\theta, \phi)$ with the corresponding SE complex beamforming vector $\mathbf{w}=\left[w_{1}, w_{2}\right]^{T}$ solved for maximum sensitivity at each scan angle.

\section{CONCLUSiOns AND RECOMMENDATIONS}

With the theoretical framework presented in this paper, single-polarized active receiving antennas can be modeled using a mixed differential and common mode analysis. The proposed mixed-mode model has been used to investigate the sensitivity of a novel active dual-mode receiving antenna utilizing, rather than rejecting, CM propagation. It was shown that, regardless of the additional noise contributed by the presence of a $\mathrm{CM}$ channel, the active dual-mode antenna exhibits only a $3 \mathrm{~dB}$ sensitivity loss at $60^{\circ} E$-plane scan, as compared to a $10 \mathrm{~dB}$ loss for a conventional active receiver rejecting $\mathrm{CM}$ propagation.

Previous works [26], [27] have shown that similar antenna structures, referred to as tripole antennas, were found to have good polarimetric beam properties and polarization discrimination capabilities over the field of view when used as a receiving antenna, which is of particular importance in radio astronomy applications [28]. Hence, the polarimetric analysis of a dual-polarized mixed differential and common mode active receiving antenna will be considered in future, as well as an array thereof operating over an increased frequency bandwidth.

\section{APPENDIX}

In addition to the MM $S$-parameters, we derive a corresponding set of $\mathrm{MM}$ equivalent noise parameters of the dLNA. Toward this end, the equivalent MM noise parameters of the balanced dLNA are expressed in terms of the SE noise parameters of the constituent SE LNAs shown in Fig. 2 (left). Note that the derivation presented here assumes that the SE LNAs are identical, isolated, and with their noise contributions uncorrelated.

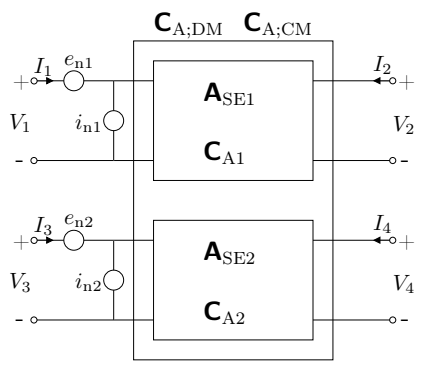

(a)

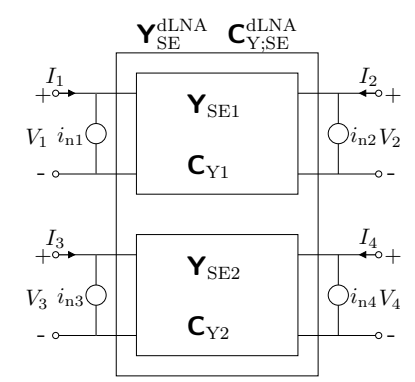

(b)
Fig. 14. Equivalent noise sources and corresponding noiseless network for two isolated LNAs in (a) chain representation, and; (b) admittance representation.

Each SE LNA can be represented by a noiseless ABCDmatrix with a noise voltage and current source applied to the input of the noiseless two-port, as shown in Fig. 14(a). This representation is referred to as the chain representation [29]. A physically significant representation of these noise sources is given by their self and cross-power spectral densities, which when arranged in matrix form constitutes the so-called noise correlation matrix, henceforth referred to as the correlation matrix. One of the fundamental advantages of the chain representation of the correlation matrix is the direct relation of its elements to the noise parameters of the two-port device, 
i.e.,

$$
\begin{aligned}
& \mathbf{C}_{\mathrm{A} 1}=\frac{1}{2}\left[\begin{array}{cc}
\left\langle e_{\mathrm{n} 1}, e_{\mathrm{n}}^{*}\right\rangle & \left\langle e_{\mathrm{n} 1}, i_{\mathrm{n} 1}^{*}\right\rangle \\
\left\langle i_{\mathrm{n} 1}, e_{\mathrm{n} 1}^{*}\right\rangle & \left\langle i_{\mathrm{n} 1}, i_{\mathrm{n} 1}^{*}\right\rangle
\end{array}\right]=\left[\begin{array}{ll}
C_{\mathrm{A} ; 11} & C_{\mathrm{A} ; 12} \\
C_{\mathrm{A} ; 21} & C_{\mathrm{A} ; 22}
\end{array}\right] \\
& =2 k_{\mathrm{B}} T_{0}\left[\begin{array}{cc}
R_{\mathrm{n}} & \frac{F_{\mathrm{min}}-1}{2}-R_{\mathrm{n}} Y_{\mathrm{opt}}^{*} \\
\frac{F_{\min }-1}{2}-R_{\mathrm{n}} Y_{\mathrm{opt}} & R_{\mathrm{n}}\left|Y_{\mathrm{opt}}\right|^{2}
\end{array}\right] \text {, }
\end{aligned}
$$

where $\left\{\left\langle e_{\mathrm{n} 1}, e_{\mathrm{n} 1}^{*}\right\rangle,\left\langle i_{\mathrm{n} 1}, i_{\mathrm{n} 1}^{*}\right\rangle\right\}$ and $\left\{\left\langle e_{\mathrm{n} 1}, i_{\mathrm{n} 1}^{*}\right\rangle,\left\langle i_{\mathrm{n} 1}, e_{\mathrm{n} 1}^{*}\right\rangle\right\}$ denote the auto- and cross-correlated spectral power densities, respectively, of the noise sources $e_{\mathrm{n} 1}$ and $i_{\mathrm{n} 1}$, and where $k_{\mathrm{B}}$ is Boltzmann's constant, $T_{0}$ is the standard temperature $(290 \mathrm{~K})$, $F_{\min }$ is the minimum noise figure, $R_{\mathrm{n}}$ is the noise resistance, and $Y_{\mathrm{opt}}$ is the optimal source admittance [29].

To derive the MM noise parameters, the chain correlation matrix is transformed to the equivalent admittance correlation matrix $\mathbf{C}_{\mathrm{Y} 1}$ using the transformation matrix $\mathbf{T}_{\mathrm{Y}}$ introduced in [29]. In the equivalent admittance representation, shown in Fig. 14(b), the LNAs are represented by their noiseless two-port admittance matrices with two noise current sources applied to the input and output ports, respectively. The admittance correlation matrix is given by

$$
\mathbf{C}_{\mathrm{Y} 1}=\mathbf{T}_{\mathrm{Y}} \mathbf{C}_{A 1} \mathbf{T}_{\mathrm{Y}}^{H},
$$

where $\mathbf{T}_{\mathrm{Y}}$ is expressed in terms of the SE admittance parameters, i.e.,

$$
\mathbf{T}_{\mathrm{Y}}=\left[\begin{array}{ll}
-Y_{\mathrm{SE} ; 11} & 1 \\
-Y_{\mathrm{SE} ; 21} & 0
\end{array}\right]
$$

Solving the admittance correlation matrix of the second LNA in a similar manner, the total admittance correlation matrix of the two uncorrelated LNAs can be formulated as

$$
\mathbf{C}_{\mathrm{Y} ; \mathrm{SE}}^{\mathrm{dLNA}}=\left[\begin{array}{c|c}
\mathbf{C}_{\mathrm{Y} 1} & \mathbf{0} \\
\hline \mathbf{0} & \mathbf{C}_{\mathrm{Y} 2}
\end{array}\right],
$$

where $\mathbf{0}$ denotes a $2 \times 2$ zero-matrix. To obtain the MM admittance correlation matrix, the transformation matrix $\mathrm{M}_{\mathrm{I}}^{\mathrm{dLNA}}$ is used, where $\mathrm{M}_{\mathrm{I}}^{\mathrm{dLNA}}$ relates the DM and CM input and output currents to the respective SE currents. That is,

$$
\mathbf{I}_{\mathrm{MM}}^{\mathrm{dLNA}}=\mathbf{M}_{\mathrm{I}}^{\mathrm{dLNA}} \mathbf{I}^{\mathrm{dLNA}},
$$

where $\mathbf{I}_{\mathrm{MM}}^{\mathrm{dLNA}}=\left[I_{\mathrm{d} 1}, I_{\mathrm{c} 1}, I_{\mathrm{d} 2}, I_{\mathrm{c} 2}\right]^{T}, \mathbf{I}^{\mathrm{dLNA}}=\left[I_{1}, I_{2}, I_{3}, I_{4}\right]^{T}$, and

$$
\mathbf{M}_{\mathrm{I}}^{\mathrm{dLNA}}=\left[\begin{array}{cccc}
\frac{1}{2} & 0 & -\frac{1}{2} & 0 \\
1 & 0 & 1 & 0 \\
0 & \frac{1}{2} & 0 & -\frac{1}{2} \\
0 & 1 & 0 & 1
\end{array}\right] .
$$

Hence, for identical LNAs, the MM admittance correlation matrix is given by

$$
\begin{aligned}
\mathbf{C}_{\mathrm{Y} ; \mathrm{MM}}^{\mathrm{dLNA}} & =\mathbf{M}_{\mathrm{I}}^{\mathrm{dLNA}} \mathbf{C}_{\mathrm{Y} ; \mathrm{SE}}^{\mathrm{dLNA}}\left(\mathbf{M}_{\mathrm{I}}^{\mathrm{dLNA}}\right)^{H} \\
& =\left[\begin{array}{cccc}
\left\langle i_{\mathrm{d} 1}, i_{\mathrm{d} 1}^{*}\right\rangle & 0 & \left\langle i_{\mathrm{d} 1}, i_{\mathrm{d} 2}^{*}\right\rangle & 0 \\
0 & \left\langle i_{\mathrm{c} 1}, i_{\mathrm{c} 1}^{*}\right\rangle & 0 & \left\langle i_{\mathrm{c} 1}, i_{\mathrm{c} 2}^{*}\right\rangle \\
\left\langle i_{\mathrm{d} 2}, i_{\mathrm{d} 1}^{*}\right\rangle & 0 & \left\langle i_{\mathrm{d} 2}, i_{\mathrm{d} 2}^{*}\right\rangle & 0 \\
0 & \left\langle i_{\mathrm{c} 2}, i_{\mathrm{c} 1}^{*}\right\rangle & 0 & \left\langle i_{\mathrm{c} 2}, i_{\mathrm{c} 2}^{*}\right\rangle
\end{array}\right] .
\end{aligned}
$$

The non-zero terms in (28) can be grouped into two equivalent DM and CM two-port admittance correlation matrices that can be related to the SE admittance correlation matrices, i.e.,

$$
\begin{gathered}
\mathbf{C}_{\mathrm{Y} ; \mathrm{DM}}=\left[\begin{array}{ll}
\left\langle i_{\mathrm{d} 1}, i_{\mathrm{d} 1}^{*}\right\rangle & \left\langle i_{\mathrm{d} 1}, i_{\mathrm{d} 2}^{*}\right\rangle \\
\left\langle i_{\mathrm{d} 2}, i_{\mathrm{d} 1}^{*}\right\rangle & \left\langle i_{\mathrm{d} 2}, i_{\mathrm{d} 2}^{*}\right\rangle
\end{array}\right]=\frac{1}{2} \mathbf{C}_{\mathrm{Y} 1} \\
\mathbf{C}_{\mathrm{Y} ; \mathrm{CM}}=\left[\begin{array}{ll}
\left\langle i_{\mathrm{c} 1}, i_{\mathrm{c} 1}^{*}\right\rangle & \left\langle i_{\mathrm{c} 1}, i_{\mathrm{c} 2}^{*}\right\rangle \\
\left\langle i_{\mathrm{c} 2}, i_{\mathrm{c} 1}^{*}\right\rangle & \left\langle i_{\mathrm{c} 2}, i_{\mathrm{c} 2}^{*}\right\rangle
\end{array}\right]=2 \mathbf{C}_{\mathrm{Y} 1} .
\end{gathered}
$$

Since the LNAs are isolated, the DM and CM admittance matrices can be expressed in terms of the SE admittance matrix $\mathbf{Y}_{\mathrm{SE}}$, where, similar to the correlation matrices, the DM and $\mathrm{CM}$ admittance matrices, respectively, result in

$$
\mathbf{Y}_{\mathrm{DM}}=\frac{1}{2} \mathbf{Y}_{\mathrm{SE}}, \quad \text { and } \quad \mathbf{Y}_{\mathrm{CM}}=2 \mathbf{Y}_{\mathrm{SE}} \text {. }
$$

The DM and CM admittance correlation matrices can now be transformed to their equivalent chain representations, after which the MM noise parameters can be solved through comparison with (22). From [29] it follows that the DM chain correlation matrix is given by the transformation

$$
\mathbf{C}_{\mathrm{A} ; \mathrm{DM}}=\mathbf{T}_{\mathrm{A} ; \mathrm{DM}} \mathbf{C}_{\mathrm{Y} ; \mathrm{DM}}\left(\mathbf{T}_{\mathrm{A} ; \mathrm{DM}}\right)^{H}
$$

where the transformation matrix $\mathbf{T}_{\mathrm{A} ; \mathrm{DM}}$ is expressed in terms of the DM ABCD-parameters:

$$
\mathbf{T}_{\mathrm{A} ; \mathrm{DM}}=\left[\begin{array}{cc}
0 & B_{\mathrm{DM}} \\
1 & D_{\mathrm{DM}}
\end{array}\right]
$$

where, on account of (30), $B_{\mathrm{DM}}=-1 / Y_{\mathrm{DM} ; 21}=-2 / Y_{\mathrm{SE} ; 21}$, and $D_{\mathrm{DM}}=-Y_{\mathrm{DM} ; 11} / Y_{\mathrm{DM} ; 21}=-Y_{\mathrm{SE} ; 11} / Y_{\mathrm{SE} ; 21}$. Substituting (23) in (29a), and then in (31), enables us to relate the elements of the DM chain correlation matrix $\mathrm{C}_{\mathrm{A} ; \mathrm{DM}}$ to the corresponding SE ones. That is, for $\mathbf{C}_{\mathrm{A} 1}=\mathbf{C}_{\mathrm{A} 2}=\mathbf{C}_{\mathrm{A}}$,

$$
\begin{aligned}
\mathbf{C}_{\mathrm{A} ; \mathrm{DM}} & =2 k_{\mathrm{B}} T_{0}\left[\begin{array}{cc}
R_{\mathrm{n}}^{\mathrm{d}} & \frac{F_{\min }^{\mathrm{d}}-1}{2}-R_{\mathrm{n}}^{\mathrm{d}}\left(Y_{\mathrm{opt}}^{\mathrm{d}}\right)^{*} \\
\frac{F_{\text {min }}^{\mathrm{d}}-1}{2}-R_{\mathrm{n}}^{\mathrm{d}} Y_{\mathrm{opt}}^{\mathrm{d}} & R_{\mathrm{n}}^{\mathrm{d}}\left|Y_{\mathrm{opt}}^{\mathrm{d}}\right|^{2}
\end{array}\right] \\
& =\left[\begin{array}{cc}
2 C_{\mathrm{A} ; 11} & C_{\mathrm{A} ; 12} \\
C_{\mathrm{A} ; 21} & \frac{1}{2} C_{\mathrm{A} ; 22}
\end{array}\right]
\end{aligned}
$$

Finally, by using (33), the DM noise parameters, and similarly the CM ones, can be expressed in terms of the standard twoport SE noise parameters $\left\{F_{\min }, R_{\mathrm{n}}, Y_{\text {opt }}\right\}$, yielding the rather intuitive result:

$$
\begin{aligned}
F_{\min }^{\mathrm{d}} & =F_{\min } & F_{\min }^{\mathrm{c}} & =F_{\min } \\
R_{\mathrm{n}}^{\mathrm{d}} & =2 R_{\mathrm{n}} & R_{\mathrm{n}}^{\mathrm{c}} & =\frac{1}{2} R_{\mathrm{n}} \\
Y_{\mathrm{opt}}^{\mathrm{d}} & =\frac{1}{2} Y_{\mathrm{opt}} & Y_{\mathrm{opt}}^{\mathrm{c}} & =2 Y_{\mathrm{opt}} .
\end{aligned}
$$

\section{REFERENCES}

[1] J. de Vaate, L. Bakker, E. E. M. Woestenburg, R. Witvers, G. Kant, and W. Van Cappellen, "Low cost low noise phased-array feeding systems for SKA pathfinders," in Antenna Technology and Applied Electromagnetics and the Canadian Radio Science Meeting, 2009. ANTEM/URSI 2009. 13th International Symposium on, Feb 2009, pp. 1-4.

[2] H. Raza, J. Yang, and M. Pantaleev, "A compact uwb passive balun solution for cryogenic 2-13 ghz eleven feed for future wideband radio telescopes," in Antennas and Propagation (EUCAP), Proceedings of the 5th European Conference on, April 2011, pp. 921-925. 
[3] L. Belostotski and J. Haslett, "A technique for differential noise figure measurement of differential LNAs," Instrumentation and Measurement, IEEE Transactions on, vol. 57, no. 7, pp. 1298-1303, July 2008.

[4] L. Tiemeijer, R. M. T. Pijper, and E. van der Heijden, "Complete onwafer noise-figure characterization of 60 -ghz differential amplifiers," Microwave Theory and Techniques, IEEE Transactions on, vol. 58, no. 6, pp. 1599-1608, June 2010.

[5] O. Perez, D. Segovia-Vargas, L. Garcia-Munoz, J. Jimenez-Martin, and V. Gonzalez-Posadas, "Broadband differential low-noise amplifier for active differential arrays," Microwave Theory and Techniques, IEEE Transactions on, vol. 59, no. 1, pp. 108-115, Jan 2011.

[6] E. De Lera Acedo, E. Garcia, V. Gonzalez-Posadas, J. Vazquez-Roy, R. Maaskant, and D. Segovia, "Study and design of a differentially-fed tapered slot antenna array," Antennas and Propagation, IEEE Transactions on, vol. 58, no. 1, pp. 68-78, Jan 2010.

[7] S. G. Hay and J. D. O"Sullivan, "Analysis of common-mode effects in a dual-polarized planar connected-array antenna," Radio Science, vol. 43, no. RS6S04, pp. 1-9, Dec. 2008.

[8] D. Cavallo, A. Neto, and G. Gerini, "Common-mode resonances in ultra wide band connected arrays of dipoles: Measurements from the demonstrator and exit strategy," in Electromagnetics in Advanced Applications, 2009. ICEAA '09. International Conference on, Sept 2009, pp. 435-438.

[9] S. Holland and M. N. Vouvakis, "The banyan tree antenna array," Antennas and Propagation, IEEE Transactions on, vol. 59, no. 11, pp. 4060-4070, Nov 2011.

[10] R. M. Page, "Monopulse radar," IRE National Conference Record, vol. 3 no. 8, pp. 132-134, 1955.

[11] P. S. Kildal, C. Orlenius, and J. Carlsson, "Ota testing in multipath of antennas and wireless devices with mimo and ofdm," Proceedings of the IEEE, vol. 100, no. 7, pp. 2145-2157, 2012.

[12] A. Hussain, P. Kildal, and A. Al-Rawi, "Efficiency, correlation, and diversity gain of uwb multiport self-grounded bow-tie antenna in rich isotropic multipath environment," in 9th International Workshop on Antenna Technology (iWAT), Karlsruhe, Germany, Mar 2013, pp. 336339.

[13] D. Prinsloo, P. Meyer, R. Maaskant, and M. Ivashina, "Design of an active dual-mode antenna with near hemispherical field of view coverage," in Electromagnetics in Advanced Applications (ICEAA), 2013 International Conference on, 2013, pp. 1064-1067.

[14] K. F. Warnick, M. V. Ivashina, and S. G. Hay, "Guest editorial for the special issue on antennas for next generation radio telescopes," Antennas and Propagation, IEEE Transactions on, vol. 59, no. 6, pp. 1786-1789, June 2011.

[15] E. E. M. Woestenburg, L. Bakker, and M. Ivashina, "Experimental results for the sensitivity of a low noise aperture array tile for the SKA," Antennas and Propagation, IEEE Transactions on, vol. 60, no. 2, pp. 915-921, Feb 2012.

[16] S. Hay, "Comparison of single-ended and differential beamforming on the efficiency of a checkerboard phased array feed in offset- and front-fed reflectors," in Antennas and Propagation (EuCAP), 2010 Proceedings of the Fourth European Conference on, April 2010, pp. $1-5$.

[17] R. Maaskant and E. E. M. Woestenburg, "Applying the active antenna impedance to achieve noise match in receiving array antennas," in Antennas and Propagation Society International Symposium, 2007 IEEE, June 2007, pp. 5889-5892.

[18] D. Bockelman and W. Eisenstadt, "Combined differential and commonmode scattering parameters: theory and simulation," Microwave Theory and Techniques, IEEE Transactions on, vol. 43, no. 7, pp. 1530-1539, Jul 1995.

[19] M. Ivashina, R. Maaskant, and B. Woestenburg, "Equivalent system representation to model the beam sensitivity of receiving antenna arrays," Antennas and Wireless Propagation Letters, IEEE, vol. 7, pp. 733-737, 2008.

[20] D. S. Prinsloo, "Characterisation of 1-band differential low noise amplifiers," Master's thesis, Stellenbosch University, Stellenbosch, South Africa, 2011.

[21] D. S. Prinsloo and P. Meyer, "Noise figure measurement of three-port differential low-noise amplifiers," IEEE Electronics Letters, vol. 48, no. 10 , pp. 578-580, May 2012.

[22] M. Robens, R. Wunderlich, and S. Heinen, "Differential noise figure deembedding: A comparison of available approaches," Microwave Theory and Techniques, IEEE Transactions on, vol. 59, no. 5, pp. 1397-1407, May 2011.

[23] R. Shaw, S. Hay, and Y. Ranga, "Development of a low-noise active balun for a dual-polarized planar connected array antenna for ASKAP," in Electromagnetics in Advanced Applications (ICEAA), 2012 International Conference on, Sept 2012, pp. 438-441.

[24] E. Jones and J. Bolljahn, "Coupled-strip-transmission-line filters and directional couplers," Microwave Theory and Techniques, IRE Transactions on, vol. 4, no. 2, pp. 75-81, 1956.

[25] M. Ivashina, O. Iupikov, R. Maaskant, W. Van Cappellen, and T. Oosterloo, "An optimal beamforming strategy for wide-field surveys with phased-array-fed reflector antennas," Antennas and Propagation, IEEE Transactions on, vol. 59, no. 6, pp. 1864-1875, June 2011.

[26] R. Karlsson, "Theory and applications of tri-axial electromagnetic field measurments," Ph.D. dissertation, Uppsala University, Uppsala, 2005. [Online]. Available: http://uu.divaportal.org/smash/record.jsf?pid=diva2:166898

[27] J. Bergman, R. Karlsson, and T. Carozzi, "System for three-dimensional evaluation," English filed as WO03/067710 A1, Aug 14, 2003.

[28] T. D. Carozzi and G. Woan, "A generalized measurement equation and van cittert-zernike theorem for wide-field radio astronomical interferometry," Monthly Notices of the Royal Astronomical Society, vol. 395, no. 3, pp. 1558-1568, May 2009.

[29] H. Hillbrand and P. Russer, "An efficient method for computer aided noise analysis of linear amplifier networks," Circuits and Systems, IEEE Transactions on, vol. 23, no. 4, pp. 235-238, Apr 1976.

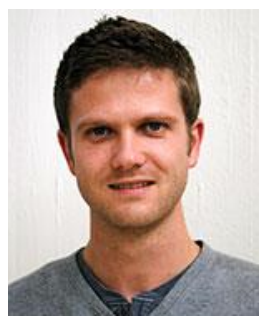

David S. Prinsloo (S'11) received the B.Eng. degree in Computer and Electronic Engineering from the Potchefstroom campus of North-West University, South Africa, in 2009 and the M.Sc.Eng. degree in Electronic Engineering from the University of Stellenbosch, Stellenbosch, South Africa in 2011. He is currently pursuing the Ph.D. degree in Electronic Engineering at the University of Stellenbosch. Since 2012 he has spent several months as a visiting researcher with the Antenna Group at the Chalmers University of Technology, Gothenburg, Sweden. His current research interests include multi-port noise theory, receiving antenna arrays, and active receiving antennas.

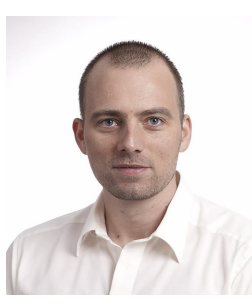

Rob Maaskant (M'11-SM'13) received his M.Sc. degree (cum laude) in 2003, and his Ph.D. degree (cum laude) in 2010, both in Electrical Engineering from the Eindhoven University of Technology, Eindhoven, The Netherlands. His Ph.D. has been awarded "the best dissertation of the Electrical Engineering Department, 2010." From 2003-2010, he was employed as an antenna research scientist at the Netherlands Institute for Radio Astronomy (ASTRON), Dwingeloo, The Netherlands, and from 2010-2012 as a postdoctoral researcher in the Antenna Group of the Signals and Systems Department at the Chalmers University of Technology, Sweden, for which he won a European Commission FP7 Marie Sklodowska-Curie Actions Outgoing - Rubicon Fellowship from the Netherlands Organization for Scientific Research (NWO), 2010. He is currently an Assistant Professor in the same Antenna Group. He is the primary author of the CAESAR software; an advanced integral-equation based solver for the analysis of large antenna array systems. His current research interest is in the field of receiving antennas for low-noise applications, meta-material based waveguides, and computational electromagnetics to solve these types of problems.

Dr. Maaskant received the 2nd best paper prize ('best team contribution') at the $2008 \mathrm{ESA} / \mathrm{ESTEC}$ workshop, Noordwijk, and was awarded a Young Researcher grant from the Swedish Research Council (VR), in 2011. He is an Associate Editor of both the IEEE Transactions on Antennas and Propagation and the FERMAT journal. 


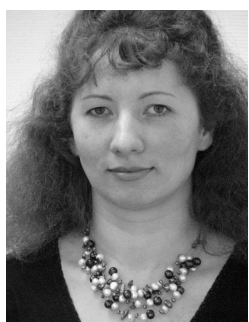

Marianna V. Ivashina (M'11-SM'13) received a Ph.D. in Electrical Engineering from the Sevastopol National Technical University (SNTU), Ukraine, in 2000. From 2001 to 2004 she was a Postdoctoral Researcher and from 2004 till 2010 an Antenna System Scientist at The Netherlands Institute for Radio Astronomy (ASTRON). During this period, she carried out research on an innovative Phased Array Feed (PAF) technology for a new-generation radio telescope, known as the Square Kilometer Array (SKA). The results of these early PAF projects have led to the definition of APERTIF - a PAF system that is being developed at ASTRON to replace the current horn feeds in the Westerbork Synthesis Radio Telescope (WSRT). Dr. Ivashina was involved in the development of APERTIF during 2008-2010 and acted as an external reviewer at the Preliminary Design Review of the Australian SKA Pathfinder (ASKAP) in 2009. In 2002, she also stayed as a Visiting Scientist with the European Space Agency (ESA), ESTEC, in the Netherlands, where she studied multiplebeam array feeds for the satellite telecommunication system Large Deployable Antenna (LDA). Dr. Ivashina received the URSI Young Scientists Award for the GA URSI, Toronto, Canada (1999), an APS/IEEE Travel Grant, Davos, Switzerland (2000), the 2nd Best Paper Award ('Best team contribution') at the ESA Antenna Workshop (2008) and the International Qualification Fellowship of the VINNOVA - Marie Curie Actions Program (2009) and The VR project grant of the Swedish Research Center (2010). She is currently an Associate Professor at the Department of Signals and Systems (Chalmers University of Technology). Her interests are wideband receiving arrays, antenna system modeling techniques, receiver noise characterization, signal processing for phased arrays, and radio astronomy. She is an Associate Editor of the IEEE Transactions on Antennas and Propagation and the FERMAT journal.

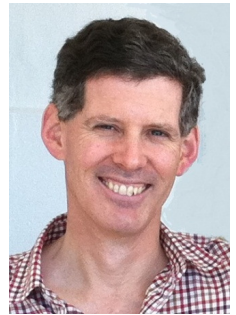

Petrie Meyer (S'87-M'88) received his Ph.D. in 1995 from the University of Stellenbosch, on numerical analysis of microstrip circuits using the Methodof-Lines. He has since worked actively in the fields of passive network design, optimization, and surrogate modelling, and has authored or co-authored more than 90 technical journal and conference papers. Since 2010 he has been involved in the design of microwave filters and ultra-low-noise amplifiers for the planned Square Kilometre Array (SKA) radio astronomy antenna, and the South-African precursor telescope, MEERKAT. In 2004, he was awarded the South African THRIP prize for human resource development, and in 2007 the international CST prize for a published journal paper making use of CST. In both 2009 and 2010, he was awarded the University of Stellenbosch Rectors award for research. He has served as chairman for the local IEEE AP/MTT conferences since 2005 and as technical chair for the 1999 IEEE Africon conference, as well as chairman of the IEEE South Africa Section during 1996-1997. He serves as regular reviewer for IEEE, IET and Wiley microwave journals. In 2009, he was elected Fellow of the South African Institute for Engineers. Since 2003 he holds the position of Professor in Microwaves and Electromagnetics at Stellenbosch University. 